\title{
An objective alternative to IUPAC's approach to assign oxidation states
}

\author{
Verònica Postils, Carlos Delgado-Alonso, Josep M. Luis, Pedro Salvador *
}

\begin{abstract}
The IUPAC has recently clarified the term Oxidation State (OS), and provided algorithms for its determination based on the ionic approximation (IA) of the bonds supported by atomic electronegativities (EN). Unfortunately, there are a number of exceptions and ambiguities in IUPAC's algorithms when it comes to practical applications. Our comprehensive study reveals the critical role of the chemical environment on establishing the OS, which cannot always be properly predicted using fix atomic EN values. By identifying what we define here as subsystems of enhanced stability within the molecular system, OS can be safely assigned in many cases without invoking exceptions. New insights about the effect of local aromaticity upon OS are revealed. Moreover, we prove that there are intrinsic limitations of the IA that cannot be overcome. In this context, the effective oxidation state (EOS) analysis arises as a robust and general scheme to derive OS without any external guidance.
\end{abstract}

Oxidation state (OS) is one of the most fundamental chemical concepts that is widely used for rationalization, categorization, and prediction of chemical reactivity of (mostly inorganic) compounds. For years, the entry of this term on IUPAC's Gold Book discussed a set of "agreed upon" rules for deriving the OS, but no formal definition was given. Consequently, a considerable debate can be found in the literature discussing misconceptions, inconsistencies or alternative OS assignment in non-trivial bonding situations over the years..$^{[1]-[4]}$ Rightly so, in 2009 the IUPAC set up a task group lead by Prof. Karen aiming at tackling the conundrum. Their conclusions were made public in 2014, with an extensive IUPAC Technical Report gathering over a hundred examples, and an essay in 2015 in this journal..[5] Final recommendations and summary of the task group were later provided, based on which the IUPAC Gold Book entries of OS and oxidation number have been most recently updated. ${ }^{[6]}$ A new generic definition for OS of an atom has been given, namely "the atom's charge after ionic approximation of its heteronuclear bonds", together with practical algorithms appropriate for molecules and solids. The IUPAC algorithm of "assigning bonds" consists in first drawing an appropriate Lewis structure and then assigning the bond electrons to the atom according to the bond ionicity. Although the authors first appeal on the molecular orbital (MO) picture, they admit that the bond ionicity should be in practice inferred from another genuine chemical concept like the electronegativity (EN) - in particular, that given by Allen's scale. ${ }^{[7]}$ For this reason, the IUPAC report is riddled with a number of ambiguities and caveats.

["] V. Postils, C. Delgado-Alonso, Dr. J. M. Luis, Dr. P. Salvador* Institut de Química Computacional i Catàlisi i Departament de Química, Universitat de Girona, Maria Aurèlia Capmany 69, 17003 , Girona, Spain

E-mail: pedro.salvador@udg.edu

Supporting information for this article is given via a link at the end of the document.
Despite the OS are intrinsically related to the electron distribution around atoms, the IUPAC reports did not discuss in depth the role of quantum-chemical calculations for OS assignment. The eventual success to ascertain formal OS from first principles has been largely hindered by the tacit assumption that partial atomic charges determine or are at least related to OS. Partial atomic charges account for the (non-integer) number of electrons that are, on average, associated to an atom by one or another partitioning scheme, while the OS is merely an integer fictitious charge. It is striking that many disputes in the literature have revolved around the association of partial atomic charges with OS ${ }^{[8]-[12]}$ (e.g. another debate concerning the OS of $\mathrm{Ti}$ in $\mathrm{TiO}_{2}$ has just sparked ${ }^{[13]}$ on the basis of partial atomic charges computed using one or another approach), while little attention has been paid to the few computational efforts going beyond this misinterpretation. ${ }^{[14],[15]}$

In 2015 some of us introduced a new and general scheme to derive OS from first principles. ${ }^{[16]}$ The effective oxidation state (EOS) method is formally applicable on equal footing to any molecular system and for any level of theory (single determinant and multireference wavefunctions) or electronic state. EOS analysis relies on the so-called effective atomic orbitals (effAOs) $)^{[17]}$, a set of distorted hybrid atomic orbitals obtained from the part of the molecule's electron density that is assigned to each atom, thus taking into account their chemical environment. Each eff-AO comes with an occupation number, that permits their identification with core/lone pairs, valence or virtual hybrids. In the EOS scheme, the eff-AOs of all atoms or fragments/ ligands ${ }^{[18]}$ are gathered for each spin case $\sigma$ (alpha or beta) and ranked in decreasing occupation number $\left(\lambda_{\mu}^{A, \sigma}\right)$ order. The first $\mathrm{n}^{\sigma}$ eff-AOs (where $\mathrm{n}^{\sigma}$ is the number of electrons) are considered occupied $\left(\lambda_{\mu}^{A, \sigma} \rightarrow 1\right)$ and the remaining empty $\left(\lambda_{\mu}^{A, \sigma} \rightarrow 0\right)$, leading to the effective configuration of the atoms or fragments/ligands within the molecule, and hence to their OS. Moreover, the larger the difference in the occupation number of the last occupied $\left(\lambda_{L O}^{\sigma}\right)$ and the first unoccupied $\left(\lambda_{F U}^{\sigma}\right)$ eff-AO, the better the electron distribution provided by the underlying wavefunction can be pictured into the discrete ionic model. As a result, a reliability index $R \equiv \min \left(R^{\alpha}, R^{\beta}\right)$ is obtained from the frontier effAOs, together with the OS assignment, defined as

$$
R^{\sigma}=100 \times \min \left(1, \lambda_{L O}^{\sigma}-\lambda_{F U}^{\sigma}+1 / 2\right) \text { where } \lambda_{L O}^{\sigma} \geq \lambda_{F U}^{\sigma},
$$

being $R=50 \%$ the worst-case scenario, with $\lambda_{L O}^{\sigma}=\lambda_{F U}^{\sigma}$. For further details of the EOS scheme and, in particular, in the case of (near) degeneracy of the frontier eff-AOs, we refer to the supporting information (SI).

The eff-AOs for the atom $A$ and spin case $\sigma(\alpha$ or $\beta$ ) are obtained as

$$
\begin{gathered}
\chi_{\mu}^{A, \sigma}(\boldsymbol{r})=\left(\lambda_{\mu}^{A, \sigma}\right)^{-1 / 2} \sum_{i}^{n^{\sigma}} U_{i \mu}^{A, \sigma} \varphi_{i}^{A, \sigma}(\boldsymbol{r}) \mu \in 1, n_{A}^{\sigma} \\
\varphi_{i}^{A, \sigma}(\boldsymbol{r}) \equiv w^{A}(\boldsymbol{r}) \varphi_{i}^{\sigma}(\boldsymbol{r})
\end{gathered}
$$

where $\lambda_{\mu}^{A, \sigma}$ is the occupation number of the $\mu$-th eff-AO, $\boldsymbol{U}^{A}$ is the unitary matrix that diagonalizes the $n^{\sigma} \times n^{\sigma}$ overlap matrix of the orbitals $\left\{\varphi_{i}^{A, \sigma}(\boldsymbol{r})\right\}$, with eigenvalues $\lambda_{\mu}^{A, \sigma},\left\{\varphi_{i}^{\sigma}(\boldsymbol{r})\right\}$ are the molecular spinorbitals and $w^{A}(\boldsymbol{r})$ is a continuous weight function that divides 
the $3 D$-space into atomic domains. For each atom $A$ there are $n_{A}^{\sigma} \leq$ $n^{\sigma}$ non-zero eigenvalues, that correspond to core/lone pairs $\left(\lambda_{\mu}^{A, \sigma} \approx\right.$ $1)$, valence $\left(\sim 0.3 \leq \lambda_{\mu}^{A, \sigma} \leq \sim 0.8\right)$ and virtual $\left(\lambda_{\mu}^{A, \sigma} \approx 0\right)$ hybrid orbitals.

Unlike other approaches, EOS analysis does not consider explicitly individual bonds or localized molecular orbitals - the wavefunction representation of a Lewis structure -, so it can tackle with no additional provisions systems with more than one dominant Lewis structure. It can also be applied to molecular and non-molecular crystals, as the eff-AOs can be obtained even in the absence of an underlying atom-centered basis set. ${ }^{[17]}$ Moreover, the EOS analysis can be easily implemented and linked to existing electronic structure codes. ${ }^{[14],[18]}$

Encouraged by the revision of the concept of OS, we have systematically assessed the performance of first-principles techniques to retrieve the OS. We have analyzed over a hundred molecular systems using the EOS method. Most of the examples have been selected for being either particularly challenging or ambiguous for the IUPAC algorithms, like Lewis and $\pi$-adducts, TM complexes with noninnocent ligands ${ }^{[20]}$ or TM carbenes. High OS TM compounds, currently in the spotlight, ${ }^{[21]}$ have also been analyzed. To assess EOS method performance, we have considered IUPAC's assignments as reference OS values, where applicable. In the case of $\pi$-adducts, reference OS are those dictated by aromaticity of the ligands, while for carbenes the textbook ionic picture of Fischer and Schrock carbenes has been assumed. Experimental spectroscopic or geometric data, when available, is in agreement with the reference OS.

Figure 1 summarizes the performance of the EOS analysis. In the majority of cases, the EOS approach predicts, without using any external guidance, OS that fully coincide with IUPAC's recipe. Where the application of the ionic approximation (IA) is ambiguous, one of the plausible OS given in the IUPAC reports or the literature is generally obtained. We pinpoint here the most illustrative examples and refer the reader to the SI for a complete account of the results, including name, formula and figures of all molecules labeled here with bold numbers in parenthesis.

\begin{tabular}{l|cc}
\hline \multicolumn{1}{c|}{ Molecular set } & \multicolumn{2}{|c}{ Agreement } \\
\hline General Examples & \multicolumn{2}{|c}{$90 \%$} \\
\hline Homonuclear bonds/clusters & \multicolumn{2}{c}{$89 \%$} \\
\hline High oxidation states & \multicolumn{2}{|c}{$100 \%$} \\
\hline Non-innocent ligands & $91 \%$ \\
\hline Lewis acid-base Adducts & \multicolumn{2}{|c}{$84 \%$} \\
\hline$\pi$-adducts & \multicolumn{2}{|c}{$93 \%$} \\
\hline Carbenes & \multicolumn{2}{|c}{}
\end{tabular}

Figure 1. Summary of the performance of EOS analysis for 101 molecular systems in comparison with IUPAC's reports [5] and previous literature.

The determination of very high oxidation states is straightforward within the IA, but it may represent a challenge for first principles analyses. In order to test the applicability of the EOS method, we have explored polyoxides and polyhydrides TM systems with formally very high OS, and identified up to $\mathrm{Os}(\mathrm{VIII})$ and $\operatorname{Ir}(\mathrm{IX})$ in $\mathrm{OsO}_{4}(\mathbf{3 0})$ and $\mathrm{IrO}_{4}{ }^{+}(\mathbf{3 1})$ species. The assignment of $\mathrm{Ti}(\mathrm{IV})$ in $\mathrm{TiO}_{2}(26)$ is undisputable, thus clearing up the doubts expressed on ref. [13]. For this particular set of systems, partial atomic charges of the TM (obtained with the same method as the eff-AOs) lie in the 1.5-2.7 range, showing no correlation with OS.

Complexes bearing non-innocent ligands are among the most difficult systems for IUPAC's algorithms because several connectivities or Lewis structures are a priori possible. Several types of non-innocent ligands have been considered with EOS method and the expected OS have been obtained in all cases. For example, EOS analysis correctly differentiates nitrosyl

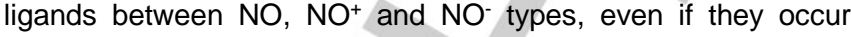
simultaneously in the same complex (e.g. $\mathrm{Fe}(\mathrm{NO})_{4}^{-}(35)$ exhibits two $\mathrm{NO}$ and two $\mathrm{NO}^{-}$ligands). Another illustrative example is the $\mathrm{Ni}\left(\mathrm{S}_{2} \mathrm{C}_{2} \mathrm{Me}_{2}\right)_{2}{ }^{\mathrm{n}-}$ series, with $\mathrm{n}=0,1,2(\mathbf{3 6}-\mathbf{3 8})$. In its most reduced form, the system is best described as a $\mathrm{Ni}(\mathrm{II})$ and two closedshell thiolate $(-2)$ ligands. In the oxidized forms the $\mathrm{Ni}$ atom retains its $\mathrm{Ni}(\mathrm{II})$ character, and the oxidation locally occurs on the thiolate ligands.

A number of dihydrogen/hydrido complexes (39-43) have also been scrutinized, being paradigmatic examples of ligand non-innocence. Hydride (-1) ligands are well identified with EOS analysis for $\operatorname{Ir}(\mathrm{III})$ and $\mathrm{Ru}(\mathrm{II})$ complexes. When $\mathrm{H}_{2}$ acts as a hapto ligand, the corresponding OS obtained is (0), as expected. For example, the EOS analysis clearly yields a $\mathrm{H}_{2}(0)$ ligand with $R=77 \%$ for the Fe complex (42), where $\mathrm{H}_{2}$ shows a typical $\mathrm{H}-\mathrm{H}$ distance of ca. $0.8 \AA$. And even if there is a significant stretching of the $\mathrm{H}-\mathrm{H}$ distance up to more than $1.6 \AA \AA^{,},{ }^{[2]}$ as in the $\mathrm{Os}(\mathrm{Cl}) \mathrm{H}_{2}(\mathrm{~L})$ complex (43) of Figure 2, the EOS analysis defining both $\mathrm{H}$ atoms on the same fragment yields a $\mathrm{H}_{2}(0)$ unit with $R=58 \%$.

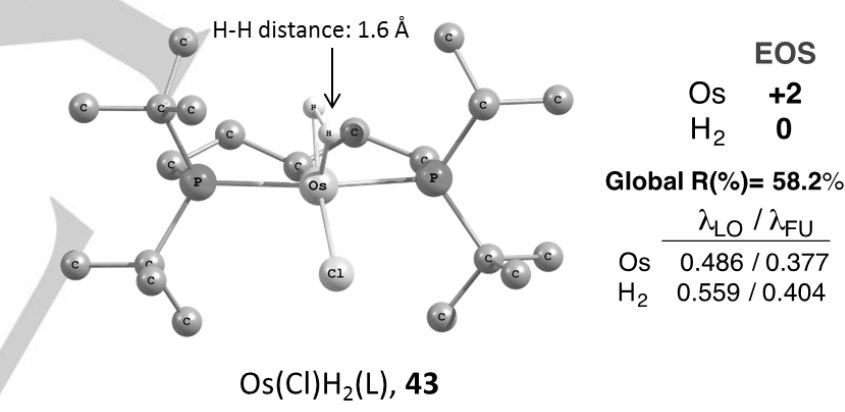

Figure 2. Representation of $\mathrm{Os}(\mathrm{Cl}) \mathrm{H}_{2}(\mathrm{~L})$ complex (43) (hydrogen atoms in the ligands omitted for clarity) and results of the EOS analysis.

The ability of IUPAC's algorithms to assign OS begins to slip noticeably when they are applied to adducts. For instance, sulfur dioxide can be found in TM complexes acting as either $Z$ type or L-type ligands, or even forming a $\pi$-complex (44-46, Figure 3). Each bonding situation requires a different strategy to derive OS within the IA. ${ }^{[5]}$ In L-type compounds, $\mathrm{SO}_{2}$ acts as a Lewis base (LB) donating two electrons to the TM center to form the bond. Since $S$ is more electronegative than $R h$, by virtue of the IA, the electron pair is assigned to $S$, which was providing it in the first place. Overall, the $\mathrm{OS}$ of the $\mathrm{SO}_{2}$ moiety is (0). However, in the Z-type case, the molecular geometry already suggests that the ligand acts as a Lewis acid (LA), thereby accepting an electron pair from the metal. The application of the IUPAC algorithm without any special provision would result in a $\mathrm{SO}_{2}$ (2-) moiety, which is at odds with the diamagnetism of the complex and the reversible binding of $\mathrm{SO}_{2}$. Then, in order to reconcile the OS assignment with experimental evidences, an exception to the IA based on Allen's EN is introduced, namely, 
when the more electronegative atom is a net acceptor of electrons, the sign of the IA must be reversed. However, there is no way to predict whether the exception must be invoked or not without an external input. On the contrary, the EOS analysis naturally yields in all cases the expected neutral $\mathrm{SO}_{2}$ ligand, no matter the bonding pattern and without any external guidance.

The expected results are also obtained with EOS analysis for a set of five adducts (47-51) where the LA is a boron atom and a lower-EN TM acts as a LB. Moreover, we have also studied three adducts where both the LA and LB are TM, for which the inherent ambiguity of the IUPAC heuristic approach is particularly notorious. While in $\mathrm{Fe}\left\{(\mathrm{CO})_{2} \mathrm{Cp}\right\}-\mathrm{Al}\left\{\left(\mathrm{C}_{6} \mathrm{H}_{5}\right)_{3}\right\}($ (52) and $\operatorname{Ir}\left\{(\mathrm{CO})_{2}\left(\mathrm{C}_{5}\left(\mathrm{CH}_{3}\right)_{5}\right)\right\}-\mathrm{W}\left\{(\mathrm{CO})_{5}\right\}(54)$ the LB is the high-EN moiety (Fe and Ir, respectively), in the analogous case of $\operatorname{Rh}\left\{(\mathrm{CO})_{2} \mathrm{Cp}\right\}-$ $\operatorname{Pt}\left\{(\mathrm{CO})\left(\mathrm{C}_{6} \mathrm{~F}_{5}\right)_{2}\right\}$ (53) the LB is the low-EN Rh moiety and the abovementioned exception must be applied. Notice that the $\mathrm{acid} / \mathrm{base}$ character of the moieties is given by the TM together with its ligands, and not merely by nature and fix EN value of the TM atom. On the other hand, the effect of the chemical environment is naturally accounted for in the WF and thus EOS is able to actually make predictions while treating all systems on equal footing.

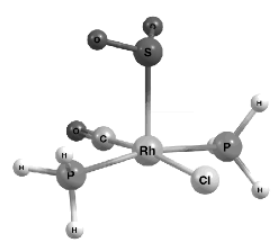

Z-type $\mathrm{SO}_{2}$ ligand $\frac{\left[\mathrm{Rh}\left(\mathrm{SO}_{2}\right) \mathrm{Cl}(\mathrm{CO})\left(\mathrm{PH}_{3}\right)_{2}\right], 44}{\mathrm{EOS}}$

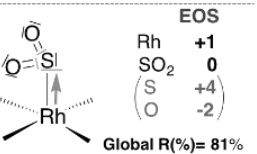
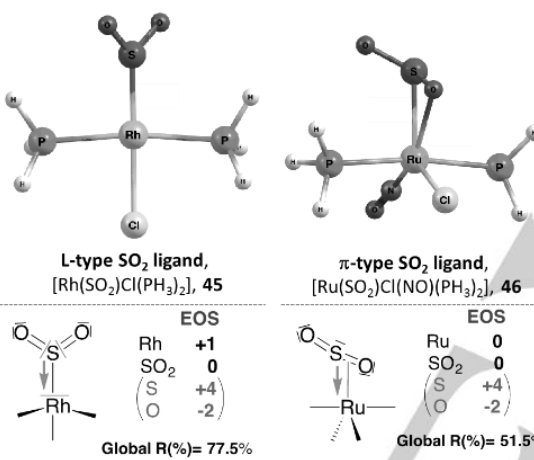
$\pi$-type $\mathrm{SO}_{2}$ ligand,
$\left[\mathrm{Ru}\left(\mathrm{SO}_{2}\right) \mathrm{Cl}\left(\mathrm{NO}^{2}\right)\left(\mathrm{PH}_{3}\right)_{2}\right], 46$

Figure 3. Binding modes of $\mathrm{SO}_{2}$ ligand and results of EOS analysis (see text).

The OS assignment of systems involving $\pi$-adducts is also particularly challenging following IUPAC's procedures, as the hapticity should be known beforehand and yet it does not uniquely determine the OS. It is the aromaticity of the $\pi$-systems that, at the end of the day, determines the OS of both the TM and the $\pi$-system. However, if the planarity of the $\pi$-system is lost -which might be difficult to foresee- the aromatic character can no longer be invoked and no clear guidelines are provided. Consequently, we have also carried out an in-depth examination of a large number of $\pi$-adducts, including 17 complexes (56-72) mentioned in the IUPAC's reports, as well as all low-lying electronic states of a series of bis(cycloheptatrienyl) TM complexes (73-86) considered by Wang et al. ${ }^{[23]}$ All $\pi$-systems with five-member rings (56-62) are readily considered by the EOS analysis as $6 \pi$ aromatic anions ( -1$)$, thus following Hückel's rule disregarding the hapticity even when a cyclopentadienyl unit is bonded to two TM (57). By the same rationale, in the case of six-membered rings, neutral benzene-like moieties would be a priori expected.

The case of the inverse-sandwich diuranium complex (64) depicted in Figure 4 is very instructive. Karen points towards an anionic (-4) $\mathrm{C}_{6} \mathrm{H}_{5} \mathrm{Me}$ moiety with $10 \pi$ electrons. ${ }^{[5]}$ Note that neutral $(0)$ with $6 \pi$ electrons would also be a plausible option. Nevertheless, there is one more element in this puzzle that has apparently gone unnoticed. The complex has a ground triplet state and careful analysis of the electron distribution reveals that the two unpaired electrons are essentially located on the $\pi$ system. Indeed, the EOS analysis assigns 5 alpha and 3 beta electrons to the $\pi$-system. That makes a total of $8 \pi$ electrons for a formal anionic $(-2) \pi$-system and two U(IV) centers. This a priori odd result turns out to be in full agreement with Baird's rule of aromaticity for triplet states. ${ }^{[24]}$ This behavior is not unique and it is also found in several $(\mathbf{7 4 , 7 6 - 7 8 )}$ of the bis(cycloheptatryenil) TM complexes analyzed. Notice that the local spin state of the $\pi$ system does not necessarily match that of the complex. For instance, $\mathrm{Mn}\left(\mathrm{C}_{7} \mathrm{H}_{7}\right)_{2}$ complex (77) is a ground-state doublet and the EOS analysis reveals that both $\mathrm{C}_{7} \mathrm{H}_{7}$ ligands are triplets antiferromagnetically coupled to a high-spin $\mathrm{Mn}$ (II) center. Thus, we can state that the local spin state of the $\pi$-systems critically determines their formal ionic charge, and consequently also the OS of the TM. Computational approaches are the only way to reveal such details of the electronic structure.

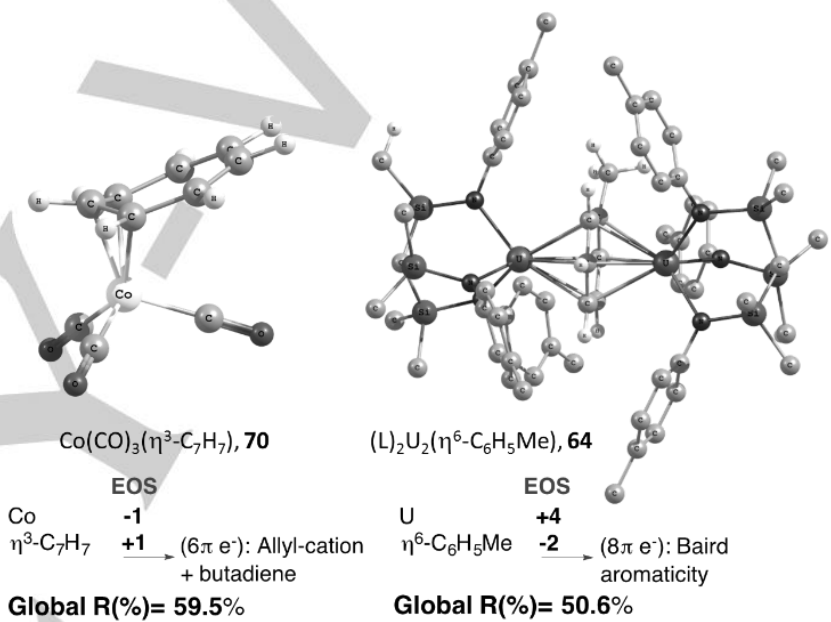

Figure 4. Sample cases of $\pi$-system ionicity driven by Baird's aromaticity (right) and conjugated segments left), and results of the EOS analysis.

In other $\pi$-adducts, the aromaticity (either Hückel or Baird) is no longer the main driving force. When the $\pi$-system is not planar, the interpretation of its ionic character should be made in terms of conjugated delocalized segments, for which the electron count is much less predictable. For instance, in $\mathrm{Co}(\mathrm{CO})_{3}\left(\eta^{3}-\mathrm{C}_{7} \mathrm{H}_{7}\right)(70$, Figure 4$)$, the ligand is clearly divided into a butadiene segment bearing two double bonds and an allyl segment coordinated to the metal. Karen assumes an allyl-anion $(-1)$ segment ${ }^{[5]}$ whereas EOS analysis predicts an allyl cation $(+1)$, for an overall $6 \pi$ bent $\mathrm{C}_{7} \mathrm{H}_{7}$ ligand. One can hardly consider either of the two options more appropriate without additional information, but we can safely claim that EOS provides the most appropriate formal ionic picture derived from its electronic distribution.

Thus far, we have seen the EOS analysis predicts without introducing exceptions the OS of the most challenging cases for the IUPAC algorithms. The results of the EOS analysis only dissent for a particular subset of systems, that basically involve main-group elements exhibiting several homonuclear bonds, like borane derivatives and homoatomic chains. Within the IA, the use of the EN criterion implies that the electrons of a homonuclear bond must always be equally divided between the atoms, whether they are symmetry-equivalent or not. We should 
bear in mind that the homonuclear bond is the worst-case scenario for the ionic model picture of the OS, as usually homonuclear bonds are highly covalent. In the language of EOS analysis, formally breaking pure homonuclear bonds results in pairs of eff-AOs belonging to different fragments with very similar occupations, which eventually (if they become the frontier eff-AOs) leads to very low values of $R$ (close to $50 \%$ ), thus blurring the OS obtained. Moreover, the results of any quantum mechanical analysis will necessarily reflect the symmetry properties of the system, so if the two atoms/fragments are not symmetry-equivalent, their OS may be different. We believe that although this issue warrants mention, the OS of this kind of systems is not important for their characterization. Conversely, since the postulated homolytic splitting does not operate in EOS analysis, a formal heterolytic homonuclear bond cleavage is possible. This is indeed the expected result for Lewis adducts where both the LA and LB moieties bear the same central atom, like for instance some unsymmetrically substituted diphosphines described in ref. [25]. In the simplest model system (55) on Figure 5, the EOS analysis clearly supports the Lewis pair picture originated from the heterolytic cleavage of the homonuclear dative bond ( $R=82 \%)$. The rationale behind this OS assignment is the formation of a $6 \pi$ aromatic phospholide anion.
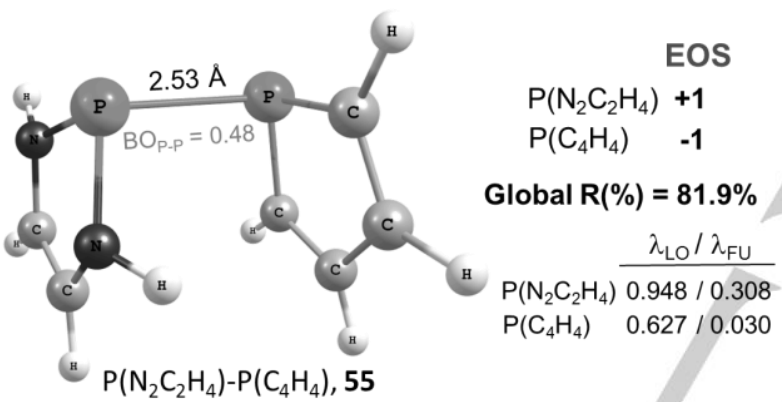

Figure 5. N-heterocyclic phosphenium-phospholide anion adduct (55) and results of the EOS analysis. P-P distance $(\AA)$ and bond order are also indicated.

Thus, most of the ambiguities and caveats of IUPAC's algorithms are overcome by first recognizing the appropriate driving force behind the adduct formation, namely the aromaticity (Hückel or Baird) or the $n$-plet stability. We introduce here the general principle of identifying subsystems of enhanced stability within the molecular system, in order to facilitate proper OS assignment. Such principle readily operates in EOS analysis as all such information is enclosed on the wavefunction.

TM carbenes challenge another key rule associated with the application of the IA, namely all electrons of the bonds between two atoms are assigned to the most EN one. We have seen many examples exhibiting formal multiple bonds where the EOS analysis nicely reproduces this rule. Yet, is there room for assigning the electrons of each bond to different atoms in a multiple bond scenario? TM carbene complexes are classified according to their reactivity as Fischer or Schrock carbenes. Radical TM carbenes have also been reported, and are best represented by a carbon-metal single bond and an additional unpaired electron sitting on the carbene fragment. From an OS perspective, the carbene moiety in Schrock carbenes is considered ionic (-2), in line with its nucleophilic character. This is readily obtained by applying the IA to the carbon-metal bonds according to the EN criterion. However, in Fisher-type systems, the carbene moiety is considered neutral $(0)$. One way to reach this OS is to assume that both the $\sigma$ and $\pi$ bonds exhibit null polarity, but this is against the general rule for resolving the IA in heteronuclear bonds. Another alternative, depicted in Figure 6, is to consider the $\sigma$ bond polarized towards the carbene and the $\pi$ bond polarized towards the metal, who keeps the electrons. This textbook picture challenges the rule that the IA equally applies to all electrons of the bonds between two atoms.

We have applied the EOS analysis to a set of prototype singlet Fisher (87-91) and Schrock (92-98) carbenes, ${ }^{[26]}$ as well as several complexes characterized as radical carbenes (99101). In six out of the seven Schrock, and in four out of five Fisher-type carbenes, the expected ionic $\mathrm{CR}_{2}(-2)$ and neutral (0) character is obtained, respectively. As no pseudodegeneracies are observed on the occupation of the effAOs, the (0) OS on the carbene fragment necessarily originates from a different splitting of the $\sigma$ and $\pi$ bonds. Visual inspection of the eff-AOs of the carbene fragment clearly supports the picture of Figure 6. Finally, the EOS analysis also reproduces the $(-1)$ OS for the carbene moiety in all radical carbenes in either singlet, doublet or triplet states.

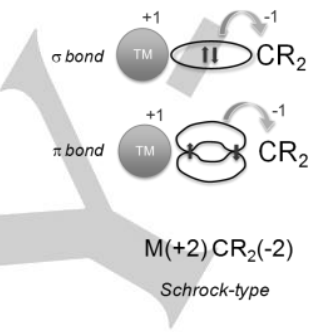

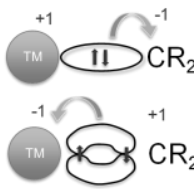

$\mathrm{M}(0) \mathrm{CR}_{2}(0)$

Fischer-type

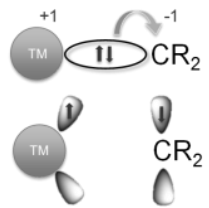

$\mathrm{M}(+1) \mathrm{CR}_{2}(-1)$

Radical (singlet)
Figure 6. Pictorial representation of singlet Schrock, Fisher and radical TMcarbene complexes and the expected OS.

To summarize, the direct application of an algorithm exclusively focused on the nature of the free atoms involved in each bond -as the IA approach supported by Allen's EN scaleprecludes the invocation of a general rule for OS assignment that accounts for all difficult cases without exceptions. The chemical environment of the atoms is an essential information that should be always explicitly used to determine the proper OS. Here we suggest the identification of subsystems of enhanced stability. However, intrinsic limitations of the IA revealed here still remain. Opportunely, the EOS method overcomes these pitfalls by not relying on Lewis structures and by properly taking into account the chemical environment encoded in the wavefunction. While in some cases EOS and IUPAC's assignment differ, we consider the former conceptually better, as it has the ability to predict its own limits of applicability by means of the values of the $R$ index. When the $R$ values are very close to $50 \%$ due to near-degeneracies on the occupations of the eff-AOs, the OS assignation should be taken with caution. The EOS analysis emerges as a general scheme to safely retrieve the OS without any external guidance.

\section{Acknowledgements}

The authors thank Dr. Eduard Matito for his helpful feedback on the manuscript. This research has been funded by the Spanish MINECO Projects CTQ2014-59212-P and CTQ2014-52525-P, 
the Catalan DIUEs 2014SGR1202 and 2014SGR931, the UdG grant GdRCompet-UdG2017 and the FEDER grant UNGI104E801. V.P. thanks the support from the Spanish MINECO BES2012-052801.

Keywords: Chemical concepts • Oxidation states • Adducts• Carbenes $\cdot$ Computational chemistry $\cdot$ Effective oxidation states

[1] J. E. M. N. Klein, B. Miehlich, M. S. Holzwarth, M. Bauer, M. Milek, M. M. Khusniyarov, G. Knizia, H.-J. Werner, B. Plietker, Angew. Chem. Int Ed. 2014, 53, 1790-1794.

[2] H. S. Yu, D. G. Truhlar, Angew. Chem. Int. Ed. 2016, 55, 9004-9006.

[3] R. Hoffmann, S. Alvarez, C. Mealli, A. Falceto, T. J. Cahill III, T. Zeng, G. Manca, Chem. Rev. 2016, 116, 8173-8192.

[4] R. Fan, J. Serrano-Plana, W. N. Oloo, A. Draksharapu, E. DelgadoPinar, A. Company, V. Martin-Diaconescu, M. Borrell, J. Lloret-Fillol, E. García-España, Y. Guo, E. L. Bominaar, L. Que, Jr., M. Costas, E. Münck, J. Am. Chem. Soc. 2018, 140, 3916-3928.

[5] a) P. Karen, P. McArdle, J. Takats, Pure Appl. Chem. 2014, 86, 1017 1081. b) ibíd 2016, 88, 831-839. c) P. Karen, Angew. Chem. Int. Ed. 2015, 54, 4716-4726.

[6] IUPAC. Compendium of Chemical Terminology, (the "Gold Book") http://goldbook.iupac.org/O04365.html.

[7] J. B. Mann, T. L. Meek, L. C. Allen. J. Am. Chem. Soc. 2000, 122, 2780-2783.

[8] A. Baranov, M. Kohout, F. R. Wagner, Y. Grin, R. Kniep, W. Bronger, Z. Anorg. Allg. Chem. 2008, 634, 2747-2753.

[9] R. Resta, Nature, 2008, 453, 735 .

[10] H. Raebiger, S. Lanny, A. Zunger, Nature, 2008, 453, 763-766.

[11] G. Aullón, S. Alvarez, Theo. Chem. Acc. 2009, 123, 67-73.

[12] M. Jansen, U. Wedig. Angew. Chem. Int. Ed. 2008, 47, 10026-10029.

[13] a) Walsh, A. A. Sokol, J. Buckeridge, D. O. Scanlon, C. R. A. Catlow, J. Phys. Chem. Lett. 2017, 8, 2074-2075. b) D. Koch, S. Manzhos, J. Phys. Chem. Lett. 2017, 8, 1593-1598.

[14] a) I. Veremchuk, T. Mori, Yu. Prots, W. Schnelle, A. Leithe-Jasper, M. Kohout, Yu. Grin, J. Solid State Chem. 2008, 181, 1983-1991. b) P. H. L. Sit, R. Car, M. R. Cohen, A. Selloni. Inorg. Chem. 2011, 50, 10259 10267. c) P. H.-L. Sit, F. Zipoli, J. Chen, R. Car, M. H. Cohen, A. Selloni, Chem. Eur. J. 2011, 17, 12136-12143. d) L. Jiang, S. V. Levchenko, A. M. Rappe, Phys. Rev. Lett. 2012, 108, 166403-5. e) G. Knizia, J. Chem. Theory Comput. 2013, 9, 4834-4843. f) J. E. M. N Klein, R. W. A. Havenith, G. Knizia, Chem. Eur. J., DOI: 10.1002/chem.201705812

[15] J. W. Thom, E. J. Sundstrom, M. Head-Gordon. Phys. Chem. Chem. Phys. 2009, 11, 11297-11304.

[16] E. Ramos-Cordoba, V. Postils, P. Salvador, J. Chem. Theory Comput. 2015, 11, 1501-1508.

[17] a) I. Mayer, J. Phys. Chem. 1996, 100, 6249-6257. b) I. Bako, A Stirling, A.P. Seitsonen, I. Mayer, Chem. Phys. Lett. 2013, 563, 97-101.

[18] E. Ramos-Cordoba, P. Salvador, I. Mayer, J. Chem. Phys. 2013, 138 214107-9.

[19] P. Salvador, E. Ramos-Cordoba, APOST-3D program (available upon request to the authors), Universitat de Girona: Girona, Spain, 2012.

[20] C. K. Jorgensen, Oxidation Numbers and Oxidation States; SpringerVerlag: New York, 1969

[21] S.X. Hu, W.-L. Li, J.-B. Lu, J. L. Bao, H. S. Yu, D. G. Truhlar, J. K. Gibson, J. Marçalo, M. Zhou, S. Riedel, W. H. E. Schwarz, J. Li, Angew. Chem. Int. Ed. 2018, 57, 3242-3245.

22] G. Gusev, A. J. Lough, Organometallics, 2002, 21, 2601-2603.

[23] H. Wang, Y. Xie, R. B. King, H. F. Schaefer, Eur. J. Inorg. Chem. 2008 , 2008, 3698-3708.

[24] Y. M. Sung, M.-C. Yoon, J. M. Lim, H. Rath, K. Naoda, A. Osuka, D. Kim, Nature Chemistry, 2015, 7, 418-422.

[25] Z. Benkő, S. Burck, D. Gudat, M. Hofmann, F. Lissner, L. Nyulászi, U. Zenneck, Chem. Eur. J. 2010, 16, 2857-2865.

[26] S. F. Vyboishchikov, G. Frenking, Chem. Eur. J. 1998, 4, 1428-1438. 


\section{COMMUNICATION}

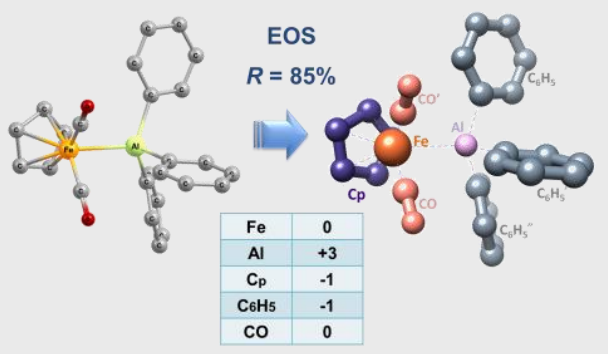

Verònica Postils, Carlos Delgado-

Alonso, Josep M. Luis, Pedro Salvador*

Page No. - Page No.

An objective alternative to IUPAC's approach to assign oxidation states 